\title{
NGHIÊN CÚUU PHÂN LOẠI HÌNH THẾ GÂY THỜI TIÊT GÂY NÓNG BÂTT THƯỜNG TRÊN KHU VỰC BẤC BỘ GIAI ĐOẠN ĐẦU MÙA ĐÔNG BẰNG PHƯƠNG PHÁP SOM
}

\author{
Võ Văn Hòa ${ }^{1}$, Dư Đức Tiến ${ }^{2}$, Trần Anh Đức², Mai Khánh Hưng², Đặng Đình Quân², \\ Nguyễn Văn Khiêm ${ }^{3}$, Nguyễn Vĩnh $\mathrm{An}^{4}$
}

Tóm tắt: Tù các số liệu quan trắc nhiệt độ trung bình ngày và nhiệt độ tối cao ngày trên khu vục Bắc Bộ trong 30 năm trở lại đây vào các tháng 11 (giai đoạn đầu mùa đông) cho thấy trung bình 2 năm xảy ra các ngày có dị thuờng nhiệt độ trung bình ngày và tối cao khá lớn (trên $5^{\circ} \mathrm{C}$ ) và tồn tại 4 đọt kéo dài hơn 2 ngày gần nhu một dạng đọt nóng (warm spell) với mức độ ở dạng gần nhu phổ biến (trên $40 \%$ số trạm ở Bắc Bộ đạt phổ biến nhiệt độ cực đại ngày tù $32^{\circ} \mathrm{C}$ đến $34^{\circ} \mathrm{C}$ ). Bài nghiên cứu sử dụng phưong pháp khai phá dĩ liệu nhiều chiều/ bản đồ tự tổ chức SOM (Self-OrganizingMaps) để phân loại các đặc trung hoàn lưu khi quyển liên quan đến hiện tuợng thời tiết nóng bất thường trên khu vực Bắc Bộ giai đoạn đầu mùa đông. So với phương pháp thần kinh nhân tạo truyền thống thi đây là mạng truyền thẳng sử dụng thuật học cạnh tranh, không giám sát. Do đó, SOM là một công cu thich hợp trong bài toán nghiên cứu và khám phá dũ liệu nhiều chiều. Sư dụng số liệu tái phân tích JRA của Nhật gồm áp suất bề mặt (liên quan đến hoạt động của vùng thấp nóng phía Tây có tâm nằm trên lãnh thổ Ân Độ - Pakistan), độ cao địa thế vị mục 500hPa (liên quan đến vai trò của Cao cận nhiệt đới Tây Bắc Thái Bình Dương) cho thấy các phân loại với 2 hoạc 3 cụm điển hình đều thấy rõ nguyên nhân chủ yếu/duy nhất liên quan đến tính dị thường của hoạt động của vùng thấp nóng phía Tây cho khu vục Bắc Bộ vào thời kì đầu mùa đông.

Từ khóa: Nóng bất thường, phương pháp SOM, đầu mùa đông, Bắc Bộ Việt Nam.

Ban Biên tập nhận bài: 12/05/2019 Ngày phản biện xong: 20/06/2019 Ngày đăng bài: 25/07/2019

\section{Mở đầu}

Phương pháp SOM được tác giả Kohonen (1989 [5], 1990 [6]) đề xuất về thực chất là một dạng của mạng thần kinh nhân tạo. Trên thực tế, từ không gian dữ liệu nhiều chiều, sau khi qua mạng SOM sẽ giảm xuống còn 1 đến 2 chiều và có thể hiển thị trên một bản đồ trong đó các đặc tính tương tự của dữ liệu có thể nhóm vào trong các nhóm tương tự nhau. Do vậy, SOM chính là công cụ hữu hiệu để phục vụ hiển thị cũng như phân tích nhóm. SOM có thể sử dụng để khám

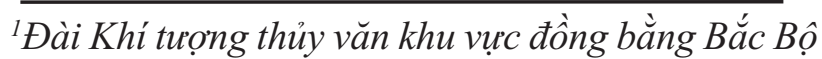
${ }^{2}$ Trung tâm Dự báo Khí tương Thủy văn quốc gia ${ }^{3}$ Văn phòng Bộ công an

${ }^{4}$ Viện Khoa học khí tượng thủy văn và Biến đổi khí hậu

Email:Vovanhoa80@gmail.com phá dữ liệu và các quan hệ giữa các dữ liệu trong tập dữ liệu nhiều chiều bằng cách đưa dữ liệu nhiều chiều về các bản đồ 2 chiều những vẫn chỉ ra được đặc tính tương tự. SOM khác với các ANN là không sử dụng các lớp ẩn (hidden layers) chỉ sử dụng input và output layer. SOM sử dụng khái niệm láng giềng (neighborhood) để giữ lại đặc trưng của các dữ liệu đầu vào trên bản đồ (có nghĩa là các training sample tương tự nhau thì được đặt gần nhau trên bản đồ). U’u điểm chính của $\mathrm{SOM}$ là biểu diễn trực quan dữ liệu nhiều chiều vào không gian ít chiều hơn và đặc trưng của dữ liệu đầu vào được giữ lại trên bản đồ.

Trong bài toán phân loại hình thế thời tiết, phương pháp SOM được ứng dụng khá phong phú, đặc biệt liên quan đến các bài toán mưa lớn, 
ví dụ ngoài nước có Koji và cộng sự (2007) [4], Liu Y và Weisberg (2005) [3], trong nước có Trần Anh Đức (2014) [1], Vũ Anh Tuấn và cộng sự (2015) [2]. Gần đây phương pháp SOM đã được ứng dụng trong bài toán phân loại hình thế gây nắng nóng, cụ thể trong bài của Seung-Yoon và cộng sự (2018) [7] đã sử dụng số liệu tái phân tích ERA-Interim của trung tâm dự báo khí tượng hạn vừa châu Âu (ECMWF) từ năm 1979 đến 2016 để phân loại hình thế đối với các ngày (xem như là nắng nóng) có nhiệt độ tối cao ngày đó lớn hơn phân vị 90 của chuỗi số liệu. Các kết quả cho thấy, các hình thế synop liên quan đến hình thành nắng nóng được phân loại thành 6 cụm dựa trên dị thường khí áp mực biển tại khu vực Đông Á.

Thông qua các số liệu quan trắc nhiệt độ trung bình ngày và nhiệt độ tối cao ngày trên khu vực Bắc Bộ trong 30 năm trở lại đây vào các tháng 11 (giai đoạn đầu mùa đông) cho thấy trung bình 2 năm xảy ra các ngày có dị thường nhiệt độ trung bình ngày và tối cao khá lớn (trên $5^{\circ} \mathrm{C}$ ) và tồn tại 4 đợt kéo dài hơn 2 ngày như các đợt nóng (warm spell) với mức độ ở dạng gần như phổ biến (trên $40 \%$ số trạm ở Bắc Bộ đạt phổ biến nhiệt độ cực đại ngày từ $32^{\circ} \mathrm{C}$ đến $34^{\circ} \mathrm{C}$ ). Dựa vào khả năng phân loại khách quan của phương pháp SOM như đã nêu, nghiên cứu sẽ thử nghiệm phân loại hình thế thời tiết điển hình liên quan đến hiện tượng dị thường thời tiết nóng vào đầu mùa đông trên khu vực Bắc Bộ. Một số đặc điểm chính của phương pháp SOM được đưa ra trong mục 2. Các kết quả và nhận xét chính được đưa ra tương ứng trong mục 3 và 4 .

\section{Phương pháp và số liệu}

\subsection{Khái quát về phưong pháp SOM}

Về kiến trúc, mạng SOM bao gồm 2 lớp: lớp tín hiệu hay là lớp đầu vào, và lớp nơ ron/Kohonen hay còn gọi là lớp đầu ra. Các nơ ron trong lớp đầu ra được sắp xếp trong một lưới 1 hoặc 2 chiều. Hình 1 minh họa bản đồ một chiều của lớp đầu ra trong đó các nơ ron được sắp xếp trong lưới một chiều. Số lượng các nơ ron trong lớp đầu vào chính là số thuộc tính của các đối tượng cần nghiên cứu. Mỗi một nơ ron trong lớp đầu vào có quan hệ truyền thẳng một chiều (feed-forward) với mỗi nơ ron trong lớp đầu ra. Điểm khác biệt trong quan hệ giữa các nơ ron trong 2 lớp của SOM so với các mạng khác là chỉ có một giá trị đầu ra cuối cùng (ví dụ như trong mạng $\mathrm{ANN}$ với $\mathrm{n}$ đầu vào có thể có $\mathrm{m}$ đầu ra). Giá trị đầu ra duy nhất này có thể đúng hoặc sai.

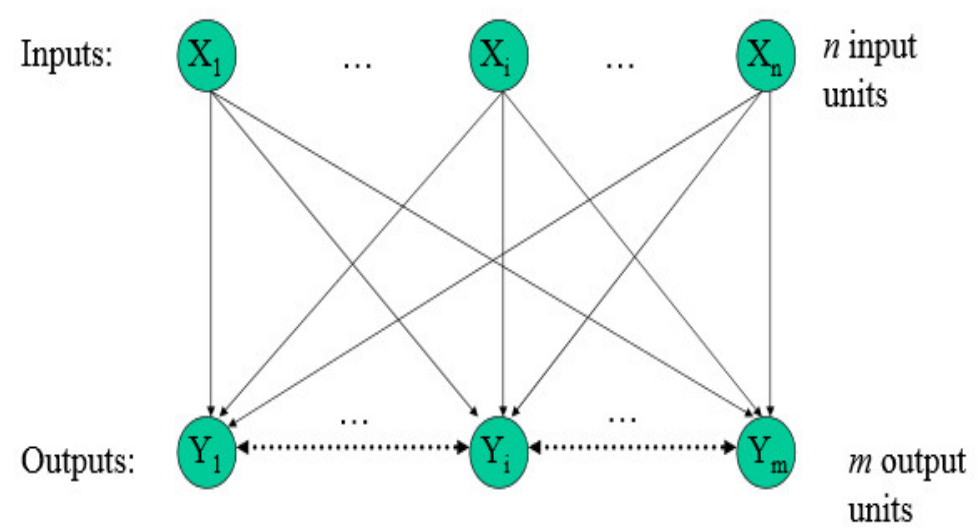

Hình 1. So đồ minh họ các lóp đầu vào và đầu ra của mạng SOM

Trong phương pháp SOM, các tín hiệu đầu vào được giải thiết là đã chuẩn hóa sao cho $\|x\|=.1$ Các tín hiệu đầu vào cho lớp đầu ra (lớp Kohonen) có thể được tính theo công thức (1) dưới đây:

$$
y_{j}=\sum_{i=1}^{n} \omega_{j i} x_{i}
$$

Trong đó $\mathrm{n}$ là số nơ ron đầu vào, $\omega_{j i}$ là trong số của nơ ron đầu vào thứ i với nơ ron đầu ra thứ j. Do trên thực tế chỉ có nơ ron đầu ra duy nhất, nên thuật toán "thắng" (winner-takes-all) được sử dụng trong phương pháp SOM. Cụ thể, nơ ron trong lớp đầu ra có giá trị $y_{j}$ lớn nhất sẽ là nơ ron thắng. 


\section{Quá trình luyện cạnh tranh:}

Gọi $x=\left(x_{1}, x_{2}, x_{3}, \ldots, x_{n}\right)^{T}$ là một đối tượng được lựa chọn ngẫu nhiên từ không gian đầu vào với $\mathrm{n}$ là kích thước mảng của không gian đầu vào. Khi đó, véc tơ trọng số của nơ ron thứ j trong mạng đầu ra sẽ là: $w_{j}=\left(\omega_{j 1}, \omega_{j 2}, \omega_{j 3}, \ldots, \omega_{j n^{*}}\right)^{T}$ với $\mathrm{n}^{*}$ là tổng số nơ ron trong lớp đầu ra. Giá trị phù hợp nhất của đối tượng đầu vào $\mathrm{x}$ với các véc tơ trọng số $w_{1}, w_{2}, w_{3}, \ldots, w_{n^{*}}$ có thể được tìm thấy bằng cách so sánh các tích số $w_{1}^{T} x, w_{2}^{T} x$, $w_{3}^{T} x, \ldots, w_{n^{*}}^{T} x$ và lựa chọn giá trị lớn nhất. Trên thực tế, tiêu chí phù hợp tốt nhất được dựa trên quá trình cực đại hóa tích số sẽ tương đương về mặt toán học với quá trình cực tiểu hóa khoảng cách Ơle (Euclidean) giữa các véc tơ $\mathrm{W}_{\mathrm{j}}$ và $\mathrm{x}$. Do đó, chỉ số $\mathrm{i}(\mathrm{x})$ của nơ ron thắng đối với đối tượng đầu vào $x$ có thể xác định qua công thức (2) dưới đây:

$$
i(x)=\arg \min _{1 \leq j \leq n^{n}}\left\|x-w_{j}\right\|
$$

\section{Quá trình hơp tác:}

Trong quá trình này, một miền lân cận hình học (topological neighborhood) được xác định sao cho nơ ron thắng được đặt tại trung tâm của miền của các nơ ron hợp tác. Gọi $h_{\mathrm{j}, t}$ là miền hợp tác có tâm tại nơ ron thắng thứ $\mathrm{t}$ và $\mathrm{d}_{\mathrm{t}, \mathrm{j}}$ là khoảng cách xung quanh giữa nơ ron thắng thứ $\mathrm{t}$ và nơ ron dễ hợp tác nhất thứ $\mathrm{j}$. Khi đó, $\mathrm{h}_{\mathrm{j}, \mathrm{t}}$ có thể là một hàm duy nhất của $\mathrm{d}_{\mathrm{t}, \mathrm{j}}$ thỏa mãn hai điều kiện như sau:

- $\mathrm{h}_{\mathrm{j}, \mathrm{t}}$ là đối xứng xung quanh điểm cực đại được xác định có $\mathrm{d}_{\mathrm{t}, \mathrm{j}}=0$.

- Biên độ của $h_{\mathrm{j}, \mathrm{t}}$ giảm đơn điệu theo sự tăng của $\mathrm{d}_{\mathrm{t}, \mathrm{j}}$ và đạt tới giá trị $0 \mathrm{khi} \mathrm{d}_{\mathrm{t}, \mathrm{j}}$ tiến tới $\infty$.

Thông thường, $\mathrm{h}_{\mathrm{j}, \mathrm{t}}$ được giải thiết tuân theo hàm phân bố Gauss như công thức (3) dưới đây:

$$
h_{j, t}=\exp \left(-\frac{d_{t, j}^{2}}{2 \sigma^{2}}\right)
$$

Trong đó $\sigma^{2}$ là một tham số đo lường mức độ nơ ron dễ hợp tác trong miền hợp tác của nơ ron thắng tham gia vào trong quá trình luyện. Trong trường hợp lưới một chiều như ở trên, khoảng cách $\mathrm{d}_{\mathrm{t}, \mathrm{j}}$ có thể xác định theo công thức (4) như sau:

$$
d_{t, j}=|t-j|
$$

Trong trường hợp lưới hai chiều, khoảng cách $\mathrm{d}_{\mathrm{t}, \mathrm{j}}$ có thể xác định theo công thức (5) dưới đây:

$$
d_{t, j}=\left\|r_{t}-r_{j}\right\|
$$

với $r_{t}$ và $r_{j}$ là các véc tơ rời rạc tương ứng xác định vị trí của nơ ron dễ hợp tác thứ j và vị trị của nơ ron thắng thứ $\mathrm{t}$.

3. Quá trình thich úng: trong quá trình này, véc tơ trọng số $\mathrm{w}_{\mathrm{j}}$ của nơ ron thứ $\mathrm{j}$ thay đổi theo đối tượng đầu vào $\mathrm{x}$. Với giá trị đưa ra của véc tơ trọng số thứ j là $w_{j}^{(s)}$ tại thời điểm $\mathrm{s}$ hoặc lần lặp $\mathrm{s}$, véc tơ trọng số mới $w_{j}^{(s+1)}$ tại thời điểm $\mathrm{s}+1$ được tính theo công thức (6):

$$
w_{j}^{(s+1)}=w_{j}^{(s)}+\eta(s) h_{j, i(x)}(s)\left(x-w_{j}^{(s)}\right)
$$

Với $\eta(s)$ là tham số đặc trưng cho tốc độ học (giảm theo hàm mũ) và xác định theo công thức (7) dưới đây:

$$
\eta(s)=\eta_{0} \exp \left(-\frac{s}{\tau_{2}}\right) \quad \mathrm{s}=0,1,2, \ldots
$$

và $h_{j, i(x)}(s)$ : là hàm lân cận được tính theo công thức (8):

$h_{j, i(x)}(s)=\exp \left(-\frac{d_{i(x), j}^{2}}{2 \sigma^{2}(s)}\right) \quad \mathrm{s}=0,1,2, .$.

với $\sigma(s)$ được tính theo công thức (9) dưới đây:

$$
\sigma(s)=\sigma_{0}\left(-\frac{s}{\tau_{1}}\right)
$$

Theo Haykin (1999) [37], các hằng số $\eta_{0}, \sigma_{0}$ $\tau_{1}$ và $\tau_{2}$ được mặc định như sau: $\eta_{0}=0.1, \sigma_{0}$ bằng bán kính của lưới, $\tau_{1}=1000 / \log \left(\sigma_{0}\right)$, $\tau_{2}=1000$

Nếu gọi $\mathrm{D}$ là tập dữ liệu đưa vào và $\mathrm{d} *$ là số chiều của bản đồ đặc tính, giải thuật (thuật toán) thực hiện của SOM bao gồm các bước như sau:

Bước 1: Ban đầu hóa các véc tơ trọng số với $\mathrm{j}=1,2, \ldots, \mathrm{d}^{*}$ bằng cách lựa chọn ngẫu nhiên các đối tượng trong $\mathrm{D}$

Bước 2: Bắt đầu lặp

Bước 3: Gán một đối tượng $x$ trong $\mathrm{D}$ với một 
giá trị xác suất cụ thể

Bước 4: Tìm nơ ron thắng $\mathrm{i}(\mathrm{x})$ tại bước thời gian/lặp s dựa trên tiêu chí khoảng cách Ơ le cực tiểu: $i(x)=\arg \min _{1 \leq j \leq d^{*}}\left\|x-w_{j}^{(s)}\right\|$

Bước 5: Cập nhật các véc tơ trọng số của tất cả các nơ ron trong lớp ra theo công thức (6)

Bước 6: Lặp lại bước 2 nếu không có sự thay đổi đáng kể nào trong bản đồ đặc tính

Bước 7: Đưa ra bản đồ đặc tính

Nói chung, chất lượng phân cụm dữ liệu của phương pháp SOM bị ảnh hưởng rất nhiều bởi quá trình lựa chọn các tham số của mạng. Các tham số này bao gồm: kích thước của bản đồ đặc tính $\left(\mathrm{d}^{*}\right)$, số lần lặp, bán kính khởi tạo $\left(\sigma_{0}\right)$, giá trị khởi tạo cho tốc độ học $(\eta), \ldots$ Trên thực tế, không có một hướng dẫn cụ thể cho việc lựa chọn các tham số này ứng với các bài toán khác nhau áp dụng SOM. Do vậy, việc "thử sai” (trial and error) là cần thiết nhằm xác định tập các giá trị thích hợp ứng với tập dữ liệu đầu vào.

Sau khi đã chuẩn hóa dữ liệu và đưa vào phân tích PCA để lựa chọn ra các thành phần chính

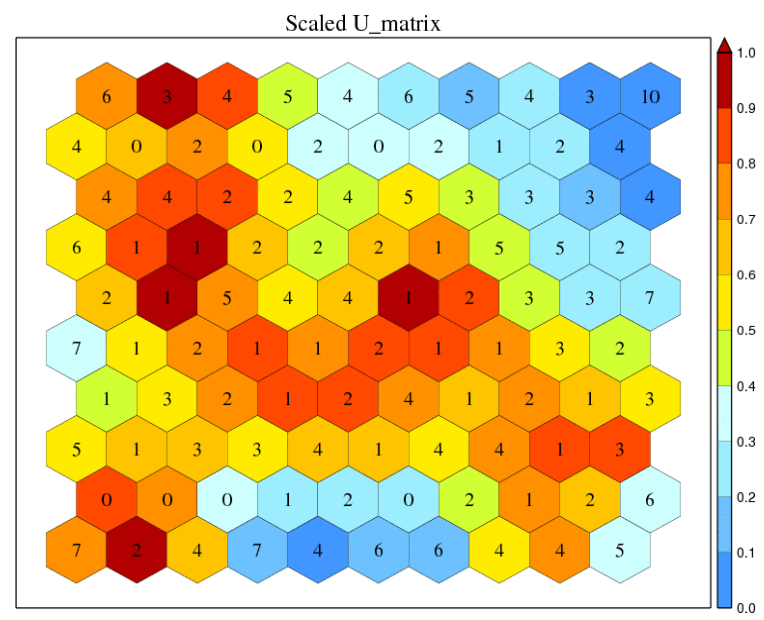

quan trọng từ tập dữ liệu ban đầu, bước tiếp theo là thực hiện luyện mạng SOM để tạo ra các bộ bản đồ tự thiếp lập/ma trận hai chiều các nơ ron Kohonen trong đó mỗi nơ ron chính là một véc tơ có kích thước bằng số nơ ron đầu vào. Như đã biết, mạng SOM có 2 dạng mạng chính là mạng hình lục giác và mạng hình vuông. Để tăng khả năng "tiếp xúc" (trao đổi thông tin theo quan hệ vật lý) giữa các nơ ron đầu ra với nhau cũng như với các nơ ron đầu vào, chúng tôi lựa chọn mạng SOM hình lục giác strong nghiên cứu này. Hình 2 dưới đây minh họa các bản đồ phân cụm dữ liệu theo mức xám được tạo ra từ $\mathrm{SOM}$ chưa được xác định ranh giới (bên trái) và đã xác định ranh giới (bên phải) được tạo ra khi thực hiện quá trình nói trên. Từ bản đồ $\mathrm{SOM}$ đã xác định ranh giới, có thể xác định được số cụm dữ liệu trong bộ số liệu đầu vào. Thông tin này sẽ được sử dụng để phân loại xem từng véc tơ dữ liệu đầu vào sẽ thuộc vào nhóm dữ liệu nào (trong nghiên cứu sử dụng phương pháp K-means).

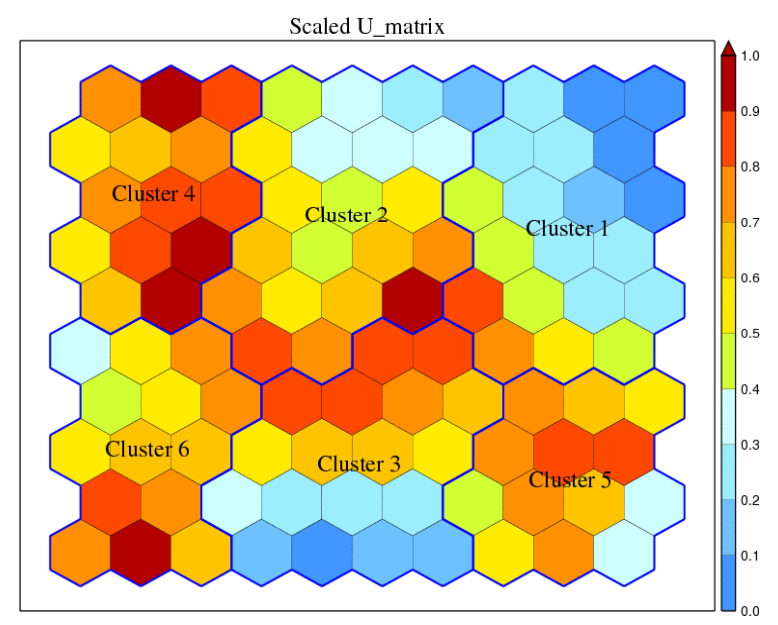

Hình 2. Vi dụ về bản đồ phân cum SOM chưa xác định ranh giới các cụm (bên trái) và đã xác định ranh giới (bên phải) để đưa vào K-Means

\subsection{Tập số liệu sử dụg}

Số liệu nhiệt độ (các phiên quan trắc, cực đại) của 82 trạm quan trắc khí tượng bề mặt trên khu vực Bắc Bộ được thu thập trong giai đoạn 1988 đến 2017 (30 năm). Phân bố theo không gian của các trạm được đưa ra trong hình 3. Để có số liệu quan trắc trên lưới phục vụ quá trình phân tích các hình thế đặc trưng khí quyển đối với hiện tượng thời tiết nóng dị thường của miền Bắc vào mùa Đông cũng như làm số liệu đầu vào cho phương pháp SOM để thiết lập ra các bản đồ phân cụm khách quan, từ đó tìm ra các nhóm hình thế synốp điển hình nghiên cứu sử dụng số liệu tái phân tích JRA25 của JMA với độ phân giải 1.25 độ. Các biến trên cao gồm trường độ cao địa thế vị $(\mathrm{H})$ và trường áp suất trung bình 
mực biển (PMSL). Thông qua thử nghiệm với dữ liệu ở các phiên phân tích $00 \mathrm{z}$ và $06 \mathrm{z}$ hoặc trung bình ngày, các kết quả tối ưu cho thấy việc sử dụng phiên phân tích $06 z$ mang lại kết quả rõ rệt và hợp lý với những cơ chế thực tế có khả năng gây ra dị thường nóng ở Bắc Bộ vào thời kì đầu mùa đông.

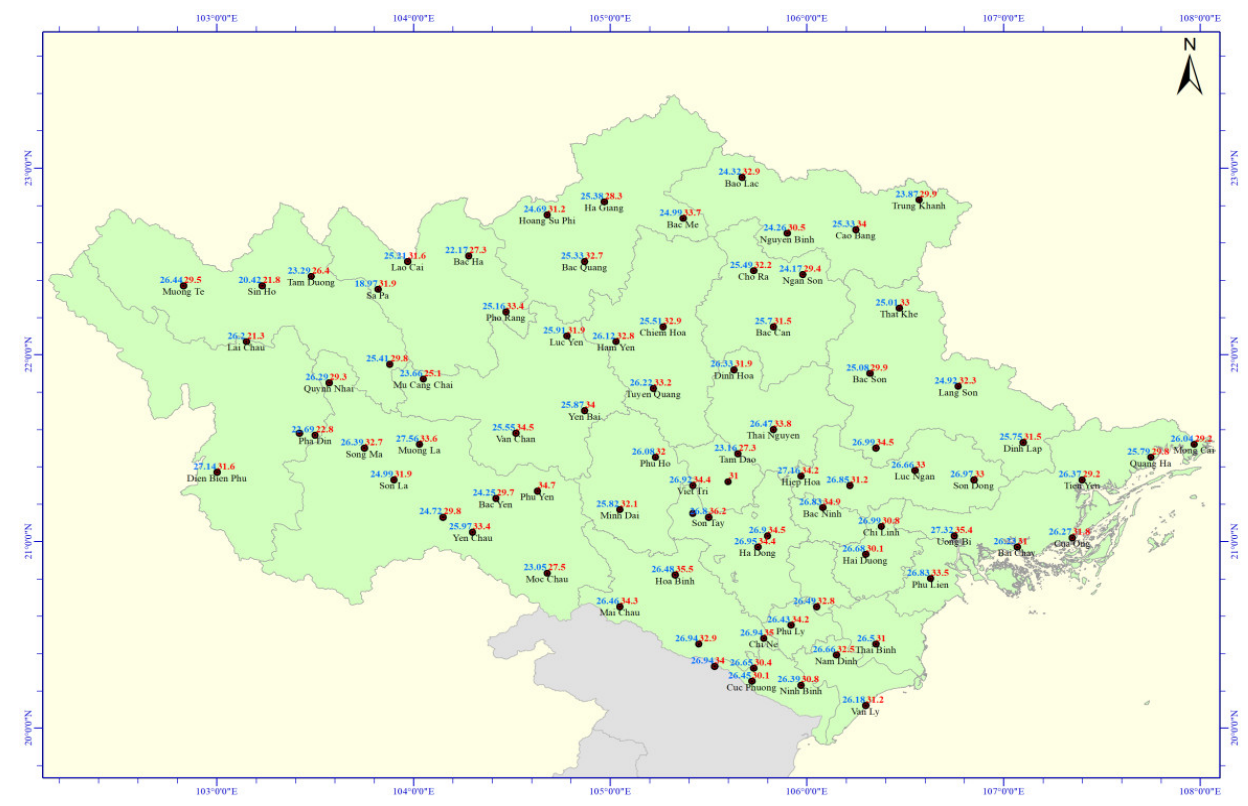

Hình 3. Nhiệt độ cưc đại ngày 17/11/2015 và nhiệt độ cực đại trung bình trong 30 năm (1989-2017) tại tùng trạm sủ dụng trong nghiên cúu

3. Kết quả phân tích khách quan bằng phương pháp SOM

Kết quả thống kê các đợt thời tiết nóng dị thường ở Bắc Bộ từ quan trắc của 82 trạm Bắc Bộ được đưa ra trong bảng 1 và 2 trong đó có cá đợt nóng dị thường điển hình như tháng 11 năm 1989, 1997, 2009 và 2015 (kéo dài ít nhất 2 ngày với Nhiệt độ tối cao ngày của các ngày có ít nhất hơn $40 \%$ số trạm trên toàn bộ Bắc Bộ (82 trạm) lớn hơn $30^{\circ} \mathrm{C}$ và 18 ngày xảy ra chỉ tiêu tương ứng (WS-1).

Theo các phân tích ở trên, các yếu tố PMSL và $\mathrm{H} 500$ trong bộ số liệu JRA25 trên lưới kinh vĩ có độ phân giải 1.25 độ bao phủ miền địa lý từ $15-50^{\circ} \mathrm{N}$ và $80-130^{\circ} \mathrm{E}$ tương ứng với $32 \times 28$ điểm nút lưới sẽ tạo ra 2x32x28=1792 biến đầu vào/nơron của lớp đầu vào trong mạng $\mathrm{SOM}$. Với số lượng biến đầu vào như này, thời gian tính toán là rất lâu và hiệu quả đem lại cũng bị giảm sút. Do đó, thay vì việc việc sử dụng trực tiếp các biến này trên lưới JRA25, chúng tôi sẽ phân tích ma trận dữ liệu này thành chuỗi những thành phần chính dựa trên kỹ thuật phân tích thành phần chính (PCA). Cụ thể, thay sử dụng 1792 biến đầu vào cho quá trình luyện phân cụm dữ liệu, thông qua PCA, chỉ cần phân cụm dữ liệu dựa trên $15-20$ thành phần chính tùy vào từng khu vực nghiên cứu.

Hình 4 và hình 5 minh hoạ cụ thể hơn dạng ma trận $\mathrm{U}$ của $\mathrm{SOM}$ ứng với từng nơ ron Kohen là trường $\mathrm{PMSL}$ và $\mathrm{H} 500$ sử dụng trong nghiên cứu thử nghiệm này. Hình 6 minh hoạ cụ thể bản đồ SOM trong trường hợp phân 2 cụm. Trong hình 4 có thể thấy rằng cựm 2 thể hiện rõ rệt việc tồn tại rõ và lâu hơn vùng thấp bao trùm được Bắc Bộ trong khi cưm 1 mức độ của vùng thấp được thể hiện yếu hơn. Sự khác biệt giữa cụm 1 và 2 trong trường $\mathrm{H} 500$ là không rõ rệt cho thấy thời kì này, vai trò của cao áp cập nhiệt là không rõ rệt trong trường hợp nghiên cứu cụ thể này. Các trường hợp kéo dài nóng dị thường từ 2-4 ngày đều được phân loại vào cụm 2 . Phân tích chi tiết hơn cho thấy trong các trường hợp này đều tồn tại một rãnh nằm trên lục địa Trung Quốc, phía trên Bắc Bộ có vai trò ngăn chặn việc xâm nhập lạnh quá sớm từ phía Bắc (cao áp Siberi) trong khi các trường hợp dị thường (mức độ vừa phải, chỉ quan trắc được một phiên dị thường) đều bị xâm nhập lạnh rất nhanh. 


\section{BÀI BÁO KHOA HỌC}

Bảng 1. Nhiệt độ tối cao ngày của các ngày có it nhất hơn 40\% số trạm trên toàn bộ Bắc Bộ lớn hon $30^{\circ} \mathrm{C}$, minh họa cho nửa Tây của Bắc Bộ (ki hiệu WS-2 ứng với hai ngày liên tiếp đạt tiêu chuẩn đã nêu)

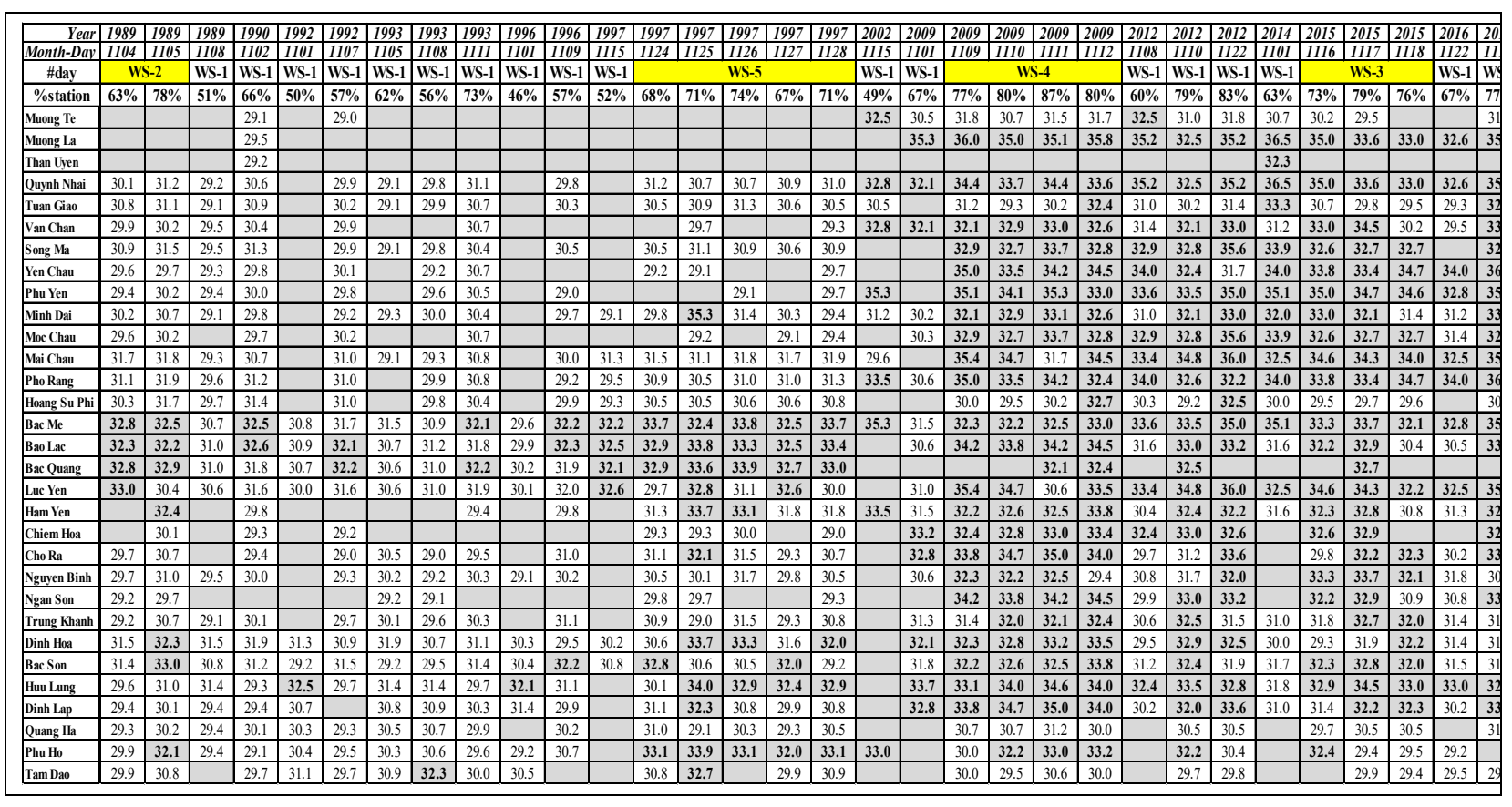

Bảng 2. Nhiệt độ tối cao ngày của các ngày có it nhất hơn 40\% số trạm trên toàn bộ Bắc Bộ lớn hơn $30^{\circ} \mathrm{C}$, minh họa cho nưa Đông của Bắc Bộ, ví dụ kí hiệu WS-2 úng với hai ngày liên tiếp đạt tiêu chuẩn đã nêu

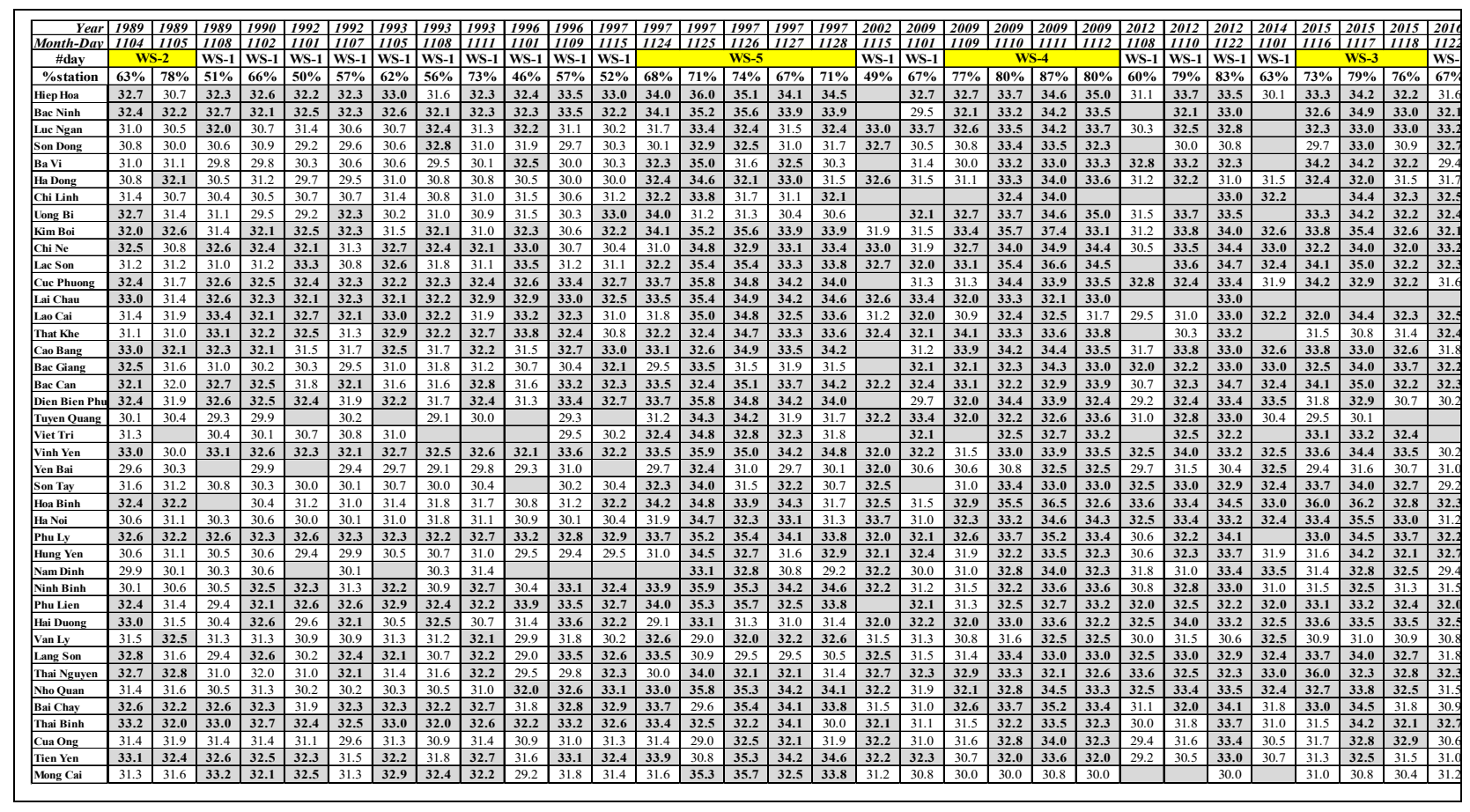




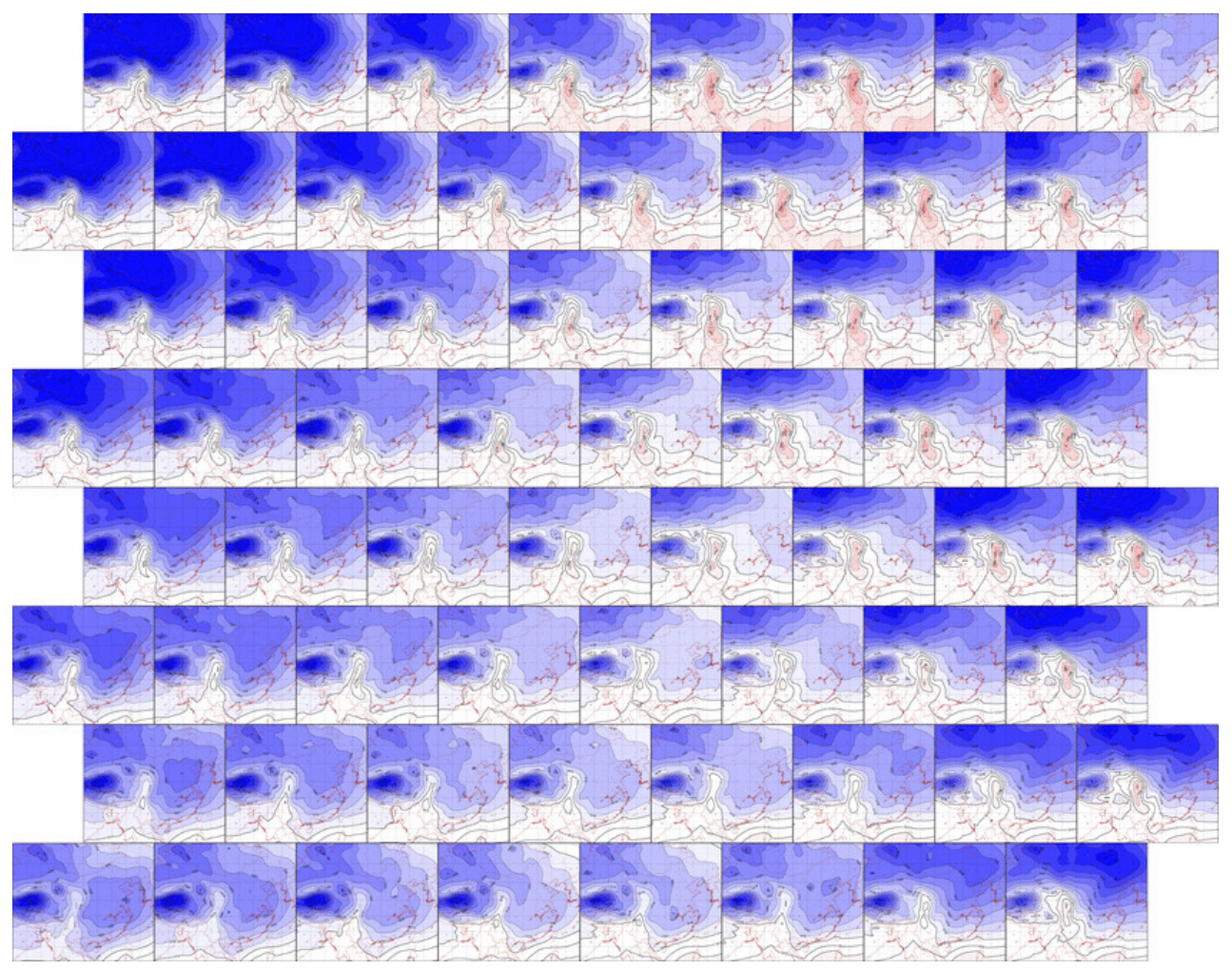

Hình 4. Minh họa các truờng áp suất bề mặt thể hiện trên ma trận SOM

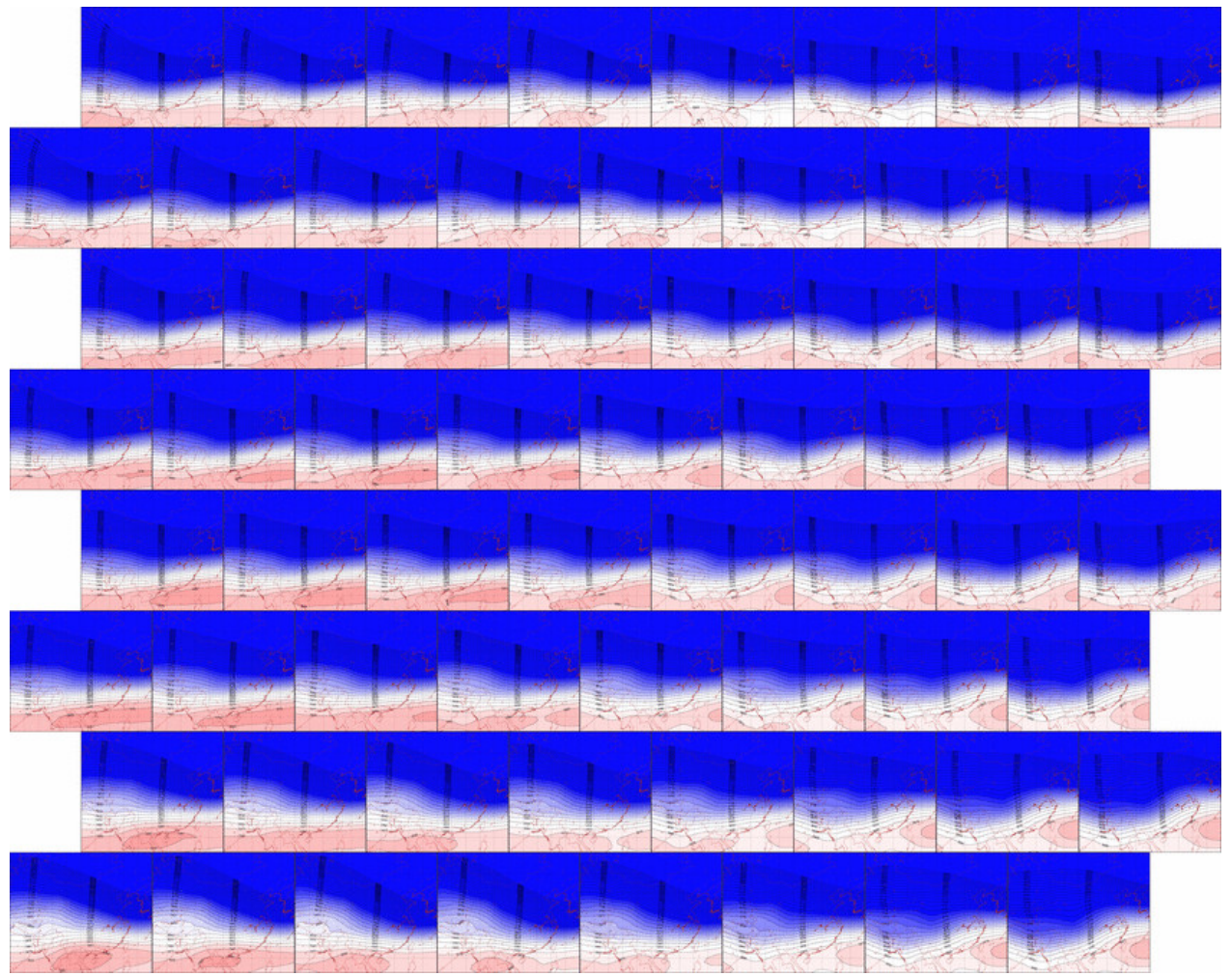

Hình 5. Minh họa các trường độ cao địa thế vị mục 500hPa thể hiện trên ma trận SOM 


\section{BÀI BÁO KHOA HỌC}
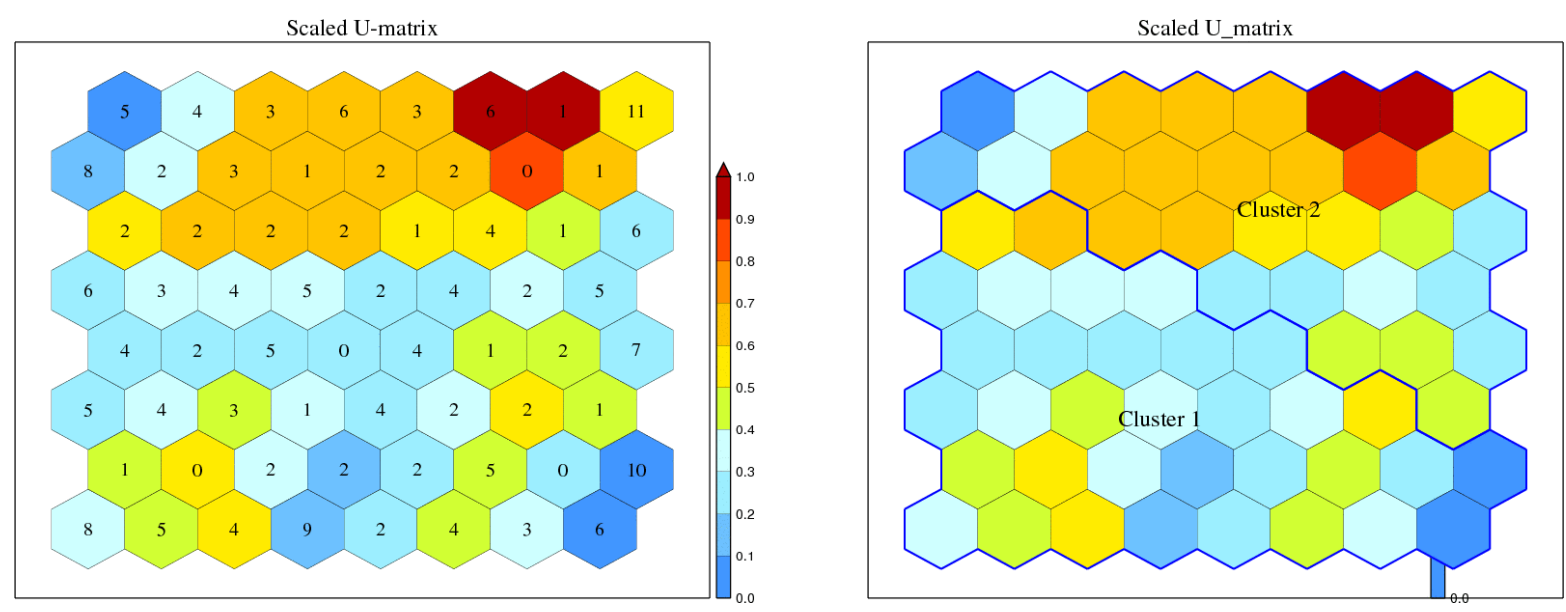

Hình 6. Ma trận SOM úng với 2 dạng hình thế co bản được phân loại đối với hiện tương nóng dị thuờng đầu mùa đông trên khu vục Bắc Bộ

\section{Kết luận}

Bài báo này đã nghiên cứu sử dụng phương pháp khai phá dữ liệu nhiều chiều SOM để phân loại các đặc trưng hoàn lưu khí quyển liên quan đến hiện tượng thời tiết nóng bất thường trên khu vực Bắc Bộ giai đoạn đầu mùa đông. Kết quả nghiên cứu ban đầu cho thấy nguyên nhân chính đều liên quan đến hoạt dộng dị thường của áp thấp nóng tồn tại trên khu vực Bắc Bộ. Việc phân 2 cự bằng phương pháp $\mathrm{SOM}$ cho thấy mức độ mở rộng của tính dị thường của vùng thấp nóng này liên quan trực tiếp đến mức độ dị thường của nhiệt độ vào đầu mùa đông trên khu vực Bắc Bộ. Trong các nghiên cứu tiếp theo chúng tôi sẽ tiếp tục thử nghiệm phân loại khách quan bằng phương pháp SOM nhưng đưa thêm vào các nhân tố thể hiện được các yếu tố tăng cường đối với hiện tượng này như gió, độ xoáy, độ ẩm ở các mực $850 \mathrm{hPa}$ liên quan đến hoạt động của rãnh trên phía Bắc.

Lời cảm ơn: Các tác giả xin gửi lời cảm ơn tới đề tài NCKH cấp Nhà nước "Nghiên cứu tác động của biến đổi khi hậu tới sự xâm nhập của các đọt lạnh và nóng ấm bất thường trong mùa đông ở khu vực miền núi phía Bắc phục vu phát triển kinh tế - xã hội”, mã số BĐKH.25/16-20 đã hỗ trợ để nhóm thực hiện nghiên cứu này. Bài báo này cũng là kết quả thực hiện của một nội dung trong đề tài nói trên.

\section{Tài liệu tham khảo}

1. Trần Anh Đức (2014), Nghiên cưu xây dựng hệ thống phân loại các hình thế gây mua lớn cho khu vưc Việt Nam bằng phương pháp SOM. Luận văn thạc sỹ ngành khí tượng khí hậu học, Đại học KHTN.

2. Vũ Anh Tuấn và cộng sự (2015), Nghiên cứu xây dụng hệ thống xác định khách quan các hình thế thời tiết gây mura lớn điển hình cho khu vực Việt Nam. Báo cáo tổng kết đề tài NCKH cấp Bộ, 179 tr.

3. Liu, Y.,Weisberg, RH. (2005), Sea surface temperature patterns on the West Florida shelf using growing hierarchical Self-Organizing Maps. J. Atmos. Ocean Tech. 23, 325-338.

4. Nishiyama, K., Endo, S., Jinno, K., Uvo, C.B., Olsson, J., Bertndtsson, R.,(2007), Idenfication of typical synoptic patterns causing heavy rainfall in the rainy season in Japan by a Self-Organizing Map. Atmospheric Research, 83, 185-200. 
5. Kohonen, T., (1989), Self-Organization and Associative Memory,3rd edition, Springer-Verlag, New York.

6. Kohonen, T., (1990), The Self-Organization map. Proceedings of the IEEE, 78(9), 1464-1480.

7. Seung-Yoon B., Kim, S.W., Jung, M.I., Roh, J.W., Son, S.W., (2018). Classification of Heat Wave Events in Seoul using Self-Organizing Map. Journal of Climate Change Research, 9, 209-221.

\author{
THE CLASSIFICATION OF TYPICAL SYNOPTIC PATTERNS \\ CAUSING ABNORMAL WARM SPELLS IN EARLY WINTER IN \\ NORTHERN AREA OF VIET NAM BY A SELF-ORGANIZING MAP \\ Vo Van Hoa', Du Duc Tien², Tran Anh Duc², Mai Khanh Hung², Dang Dinh Quan², \\ Nguyen Van Khiem ${ }^{3}$, Nguyen Vinh $\mathrm{An}^{4}$ \\ ${ }^{1}$ Red-river Delta Regional Hydro-Meteorological Center \\ ${ }^{2}$ National Center for Hydro-Meteorological Forecasting \\ ${ }^{3}$ Ministry of Public Security Office \\ ${ }^{4}$ Viet Nam Institude of Meteorology, Hydrology and Climate Change
}

\begin{abstract}
According to the observed data of daily average temperature and daily maximum temperature in the Northern region of Viet Nam in the past 30 years in November (early winter month), it shows that on average, 2 years occurred the days thatthe dailyaverage and maximum temperature anomalies are quite large (above $5^{\circ} \mathrm{C}$ ) and there are 4 warm spells lasting more than 2 days, almost like a warm spell with the degree in the almost common event (over 40\% of the stations in the Northern region reaches the maximum daily temperature from $32^{\circ} \mathrm{C}$ to $34^{\circ} \mathrm{C}$ ). The paper uses the Self-Organizing-Maps (SOM) method to classify atmospheric circulation characteristics related to abnormal warm spells in the northern region in the early winter. Compared with the artificial neural network method, this is a straight transmission network using competitive, unattended technology. Therefore, SOM is an appropriate tool in the multi-dimensional data research and exploration problem. Using JRA re-analysis data including pressure of mean sea level (related to the activities of the western hot low pressure centered on India-Pakistan territory), geopotential height of 500hPa pressure level (relative to the role of the Western Pacific Subtropical High Pressure), the classification results based on SOM shows 2 or 3 typical clusters in which it is clear that the main and unique cause related to the anomalies in activity of the western hot low pressure in the early winter months.
\end{abstract}

Keywords: Abnormal warm spell, Self-Organizing Map, early winter, northern area of Viet Nam. 